\title{
MINIMAL COLORING NUMBERS ON MINIMAL DIAGRAMS OF TORUS LINKS
}

\author{
KAZUHIRO ICHIHARA, KATSUMI ISHIKAWA, AND ERI MATSUDO
}

\begin{abstract}
We determine the minimal number of colors for non-trivial $\mathbb{Z}$ colorings on the standard minimal diagrams of $\mathbb{Z}$-colorable torus links. Also included are complete classifications of such $\mathbb{Z}$-colorings and of such $\mathbb{Z}$-colorings by only four colors, which are shown by using rack colorings on link diagrams.
\end{abstract}

\section{INTRODUCTION}

This is a continuation of the study of $\mathbb{Z}$-colorings on the standard minimal diagrams of $\mathbb{Z}$-colorable torus links given in [6. Section 3] by the first and the third named authors.

Previously, in [5], as a generalization to the well-known Fox's coloring originally introduced in [3], they defined a $\mathbb{Z}$-coloring for a link in the 3 -space as follows. A map $\gamma:\{\operatorname{arcs}$ of $D\} \rightarrow \mathbb{Z}$ for a regular diagram $D$ of a link is called a $\mathbb{Z}$-coloring if it satisfies the condition $2 \gamma(a)=\gamma(b)+\gamma(c)$ at each crossing of $D$ with the over arc $a$ and the under arcs $b$ and $c$. We say that a link is $\mathbb{Z}$-colorable if it has a diagram admitting a non-trivial $\mathbb{Z}$-coloring, i.e., there are at least two distinct colors on the diagram. We remark that a link $L$ is $\mathbb{Z}$-colorable if and only if the determinant $\operatorname{det}(L)$ of $L$ equals 0 . Since the determinant of any knot (single component link) is shown to be an odd integer, any knot is not $\mathbb{Z}$-colorable.

There are several studies on the minimal coloring number (i.e., the minimal number of the colors) of Fox colorings on knots and links; some upper and lower bounds have been obtained. In the same line, it was shown in [5. Theorem 3.1] that the minimal coloring number $\operatorname{mincol}_{\mathbb{Z}}(L)$ of a non-splittable $\mathbb{Z}$-colorable link $L$ is at least four. (Note that $\operatorname{mincol}_{\mathbb{Z}}(L)=2$ for any splittable link $L$.) However, in contrast to the case of the Fox coloring, it was proved that $\operatorname{mincol}_{\mathbb{Z}}(L)=4$ for any non-splittable $\mathbb{Z}$-colorable link $L$, by the third author in [8], and independently by Meiqiao Zhang, Xian'an Jin, and Qingying Deng in [10.

Thereafter, in [6], the first and third authors consider the minimal coloring number $\operatorname{mincol}_{\mathbb{Z}}(D)$ of a minimal diagram $D$ of a $\mathbb{Z}$-colorable link. (A minimal diagram is a diagram representing the link with least number of crossings.)

In particular, in [6, Section 3], they consider torus links, that is, the links which can be isotoped onto the standardly embedded torus in the 3-space. By $T(a, b)$, we mean the torus link running $a$ times meridionally and $b$ times longitudinally. It is known that $T(a, b)$ is $\mathbb{Z}$-colorable if $a$ or $b$ is even. Actually, it is shown in

Date: August 5, 2019.

2010 Mathematics Subject Classification. 57M25.

Key words and phrases. $\mathbb{Z}$-coloring, torus link, minimal diagram.

The first author is partially supported by JSPS KAKENHI Grant Number JP18K03287.

The second author was partially supported by JSPS KAKENHI Grant Number JP16J01183. 
6. Theorem 1.3] that $\operatorname{mincol}_{\mathbb{Z}}(D)=4$ for the standard diagram $D$ of $T(p n, n)$ illustrated by Figure 1 with $n>2$, even and $p \neq 0$. Extending this, in this paper, we show the following:

Theorem 1.1. Let $p, q$, and $r$ be non-zero integers such that $p$ and $q$ are relatively prime, $|p| \geq q \geq 1$, and $r \geq 2$. Let $D$ be the standard diagram of $T(p r, q r)$ illustrated by Figure 1. Suppose that T $(p r, q r)$ is $\mathbb{Z}$-colorable, or, equivalently, pr or qr is even. Then, mincol $_{\mathbb{Z}}(D)=4$ if $r$ is even, and $\operatorname{mincol}_{\mathbb{Z}}(D)=5$ if $r$ is odd.

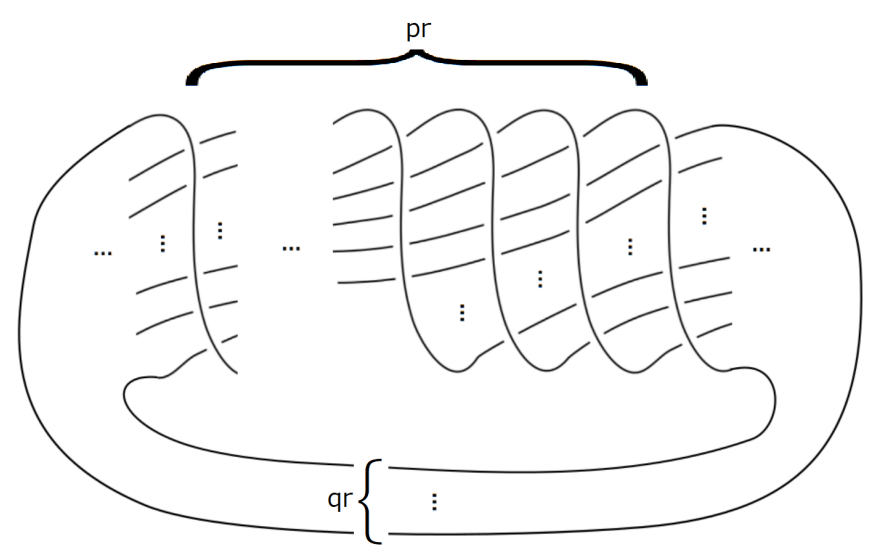

FiguRE 1. The standard diagram of $T(p r, q r)$

We remark that the diagram $D$ has the least number of crossings for the torus link. See [4 for example. Also if $r=1$, then the link becomes a knot, which is not $\mathbb{Z}$-colorable. (Actually $r$ coincides with the number of components of the torus link.) The theorem above is proved in Section 2 (when $r$ is even) and Section 3 (when $r$ is odd).

We also include complete classifications of all the $\mathbb{Z}$-colorings on the standard diagram of $T(a, b)$ (Proposition 4.3) and of all the $\mathbb{Z}$-colorings by only four colors of $T(a, b)$ (Proposition 4.4) in Section 4. The key of our proof of the propositions is to use rack colorings on link diagrams. A theorem used to prove the propositions, which can be of interest independently, is proved in Appendix. That part is essentially based on the master thesis [7] of the second author.

\section{FOUR COLORS CASE}

In this section, we prove the following, showing the first assertion of Theorem 1.1 .

Theorem 2.1. Let $p, q$, and $r$ be non-zero integers such that $p$ and $q$ are relatively prime, $|p| \geq q \geq 1$, and $r \geq 2$. Let $D$ be the standard diagram of $T(p r, q r)$ illustrated by Figure 1. Suppose that $T(p r, q r)$ is $\mathbb{Z}$-colorable, or, equivalently, pr or qr is even. Then, $\operatorname{mincol}_{\mathbb{Z}}(D)=4$ if $r$ is even.

Proof. We will find a $\mathbb{Z}$-coloring $\gamma$ with only four colors on $D$ by assigning colors on the $\operatorname{arcs}$ of $D$.

Note that the link has $r$ components each of which runs longitudinally $q$ times and twists meridionally $p$ times as shown in Figure 1. In a local view, we see $q r$ 
horizontal parallel arcs in $D$. We divide such arcs into $q$ subfamilies $\mathbf{x}_{1}, \ldots, \mathbf{x}_{q}$ as depicted in Figure 2 (left).
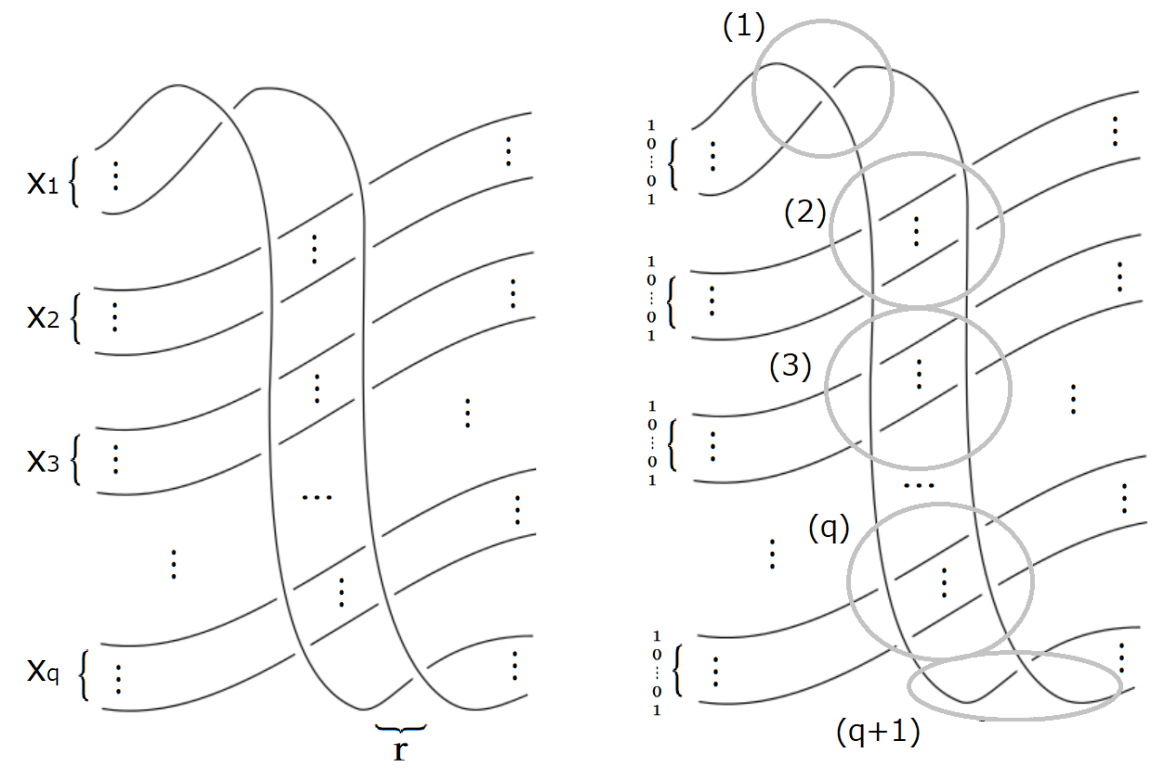

FiguRE 2.

We first find a local $\mathbb{Z}$-coloring $\gamma$ on the local diagram shown in Figure 2 (left). Let us start with setting $\left(\gamma\left(x_{i, 1}\right), \gamma\left(x_{i, 2}\right), \ldots, \gamma\left(x_{i, r}\right)\right)=(1,0, \ldots, 0,1)$ for any $i$. See Figure 2 (right).

Since $r$ is assumed to be even, as illustrated in Figures 3 (left), we can extend $\gamma$ on the arcs in the regions $(1)$ and $(q+1)$ in Figure 2 (right), and, as illustrated in Figures 3 (right), we can extend $\gamma$ on the arcs in the regions $(2),(3), \ldots,(q)$ in Figure 2 (right). Thus $\gamma$ can be extended on all the arcs in the local diagram as depicted in Figure 4.
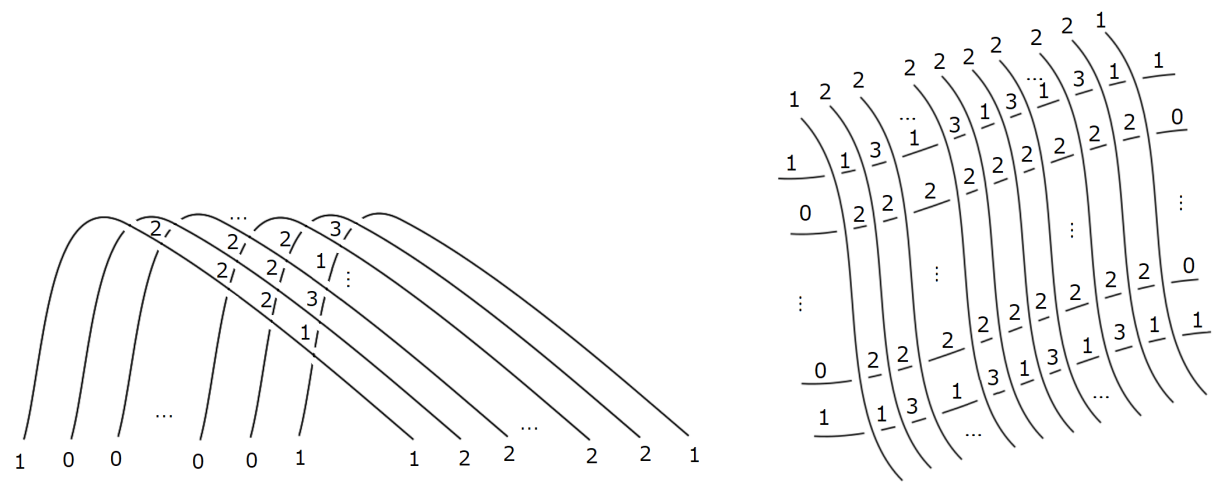

Figure 3. 


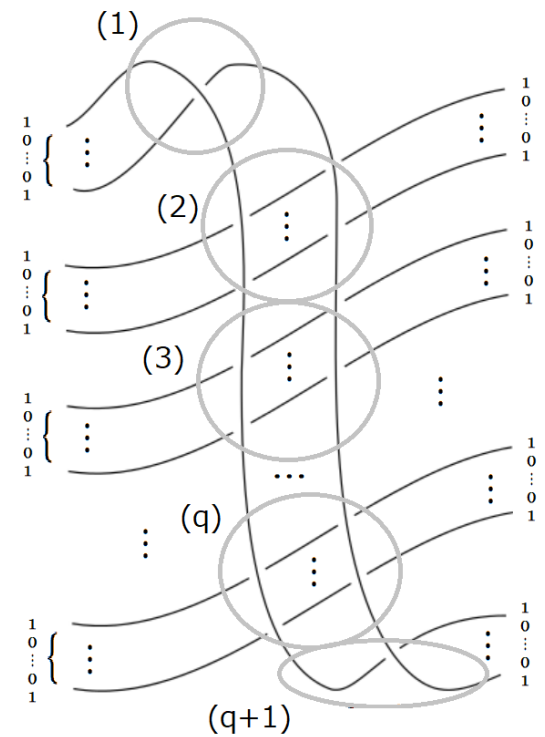

FIGURE 4.

Since $D$ is composed of $p$ copies of the local diagram in Figure 2 (left), it concludes that $D$ admits a $\mathbb{Z}$-coloring with only four colors $0,1,2$, and 3 .

Since the torus link is non-splittable, $\operatorname{mincol}_{\mathbb{Z}}(D)$ must be at least four by $[5$, Theorem 3.1], and we conclude that $\operatorname{mincol}_{\mathbb{Z}}(D)=4$.

\section{Five COLORS CASE}

In this section, we prove the following, which asserts the second half of the statement of Theorem 1.1 .

Theorem 3.1. Let $p, q$, and $r$ be non-zero integers such that $p$ and $q$ are relatively prime, $|p| \geq q \geq 1$, and $r \geq 2$. Let $D$ be the standard diagram of $T(p r, q r)$ illustrated by Figure 1. Suppose that $T(p r, q r)$ is $\mathbb{Z}$-colorable, or, equivalently, pr or qr is even. Then $\operatorname{mincol}_{\mathbb{Z}}(D)=5$ if $r$ is odd.

To prove this, we recall the following lemma, which was obtained in [5].

Lemma 3.2. For $a \mathbb{Z}$-coloring $\gamma$ with $0=\min \operatorname{Im} \gamma$, if an over arc at a crossing is colored by 0 , then the under arcs at the crossing are colored by 0 . For a $\mathbb{Z}$-coloring $\gamma$ with $M=\max \operatorname{Im} \gamma$, if an over arc at a crossing is colored by $M$, then the under arcs at the crossing are colored by $M$.

Proof of Theorem 3.1. We first show that the minimal coloring number $\operatorname{mincol}_{\mathbb{Z}}(D)$ of $D$ is at most five, i.e., $\operatorname{mincol}_{\mathbb{Z}}(D) \leq 5$.

In the following, we will find a $\mathbb{Z}$-coloring $\gamma$ with five colors on $D$ by assigning colors on the $\operatorname{arcs}$ of $D$. In the same way as in the proof of Theorem 2.1. we find a local $\mathbb{Z}$-coloring $\gamma$ on the local diagram shown in Figure 2 (left), and extend it to whole the diagram. Note that if $r$ is odd and $T(p r, q r)$ is $\mathbb{Z}$-colorable, then either $p$ or $q$ must be even. 
First, suppose that $p$ is even. In this case, we start with setting $\left(\gamma\left(x_{i, 1}\right), \gamma\left(x_{i, 2}\right), \ldots, \gamma\left(x_{i, r}\right)\right)=$ $(1,0, \ldots, 0,1)$ for any $i$. See Figure 2 (right) again. As illustrated in Figure 5 (left) and (right), we can extend $\gamma$ on the arcs in the regions $(1)$ and $(q+1)$ in Figure 2 (right).
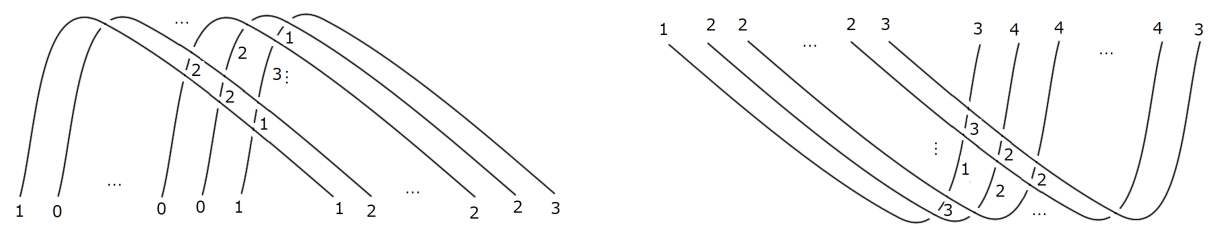

FIGURE 5 .

As illustrated in Figure 6 (left), we can extend $\gamma$ on the arcs in the regions $(2),(3), \ldots,(q)$ in Figure 2 (right). Then, as shown in Figure 6 (right), we can extend $\gamma$ to all the arcs in Figure 2 (right).
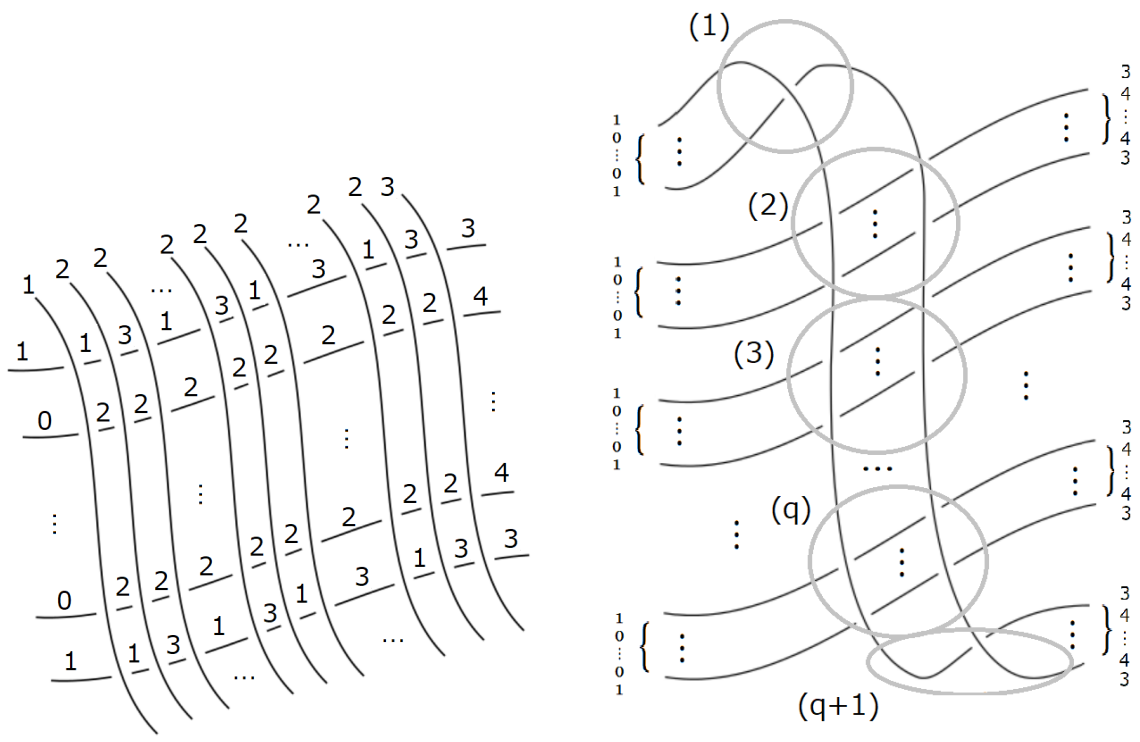

FiguRE 6.

Without contradicting to the condition of the coloring, we can connect the local diagram in Figure 6 (right) with the image of $\pi$-rotation. See Figure 7 , 


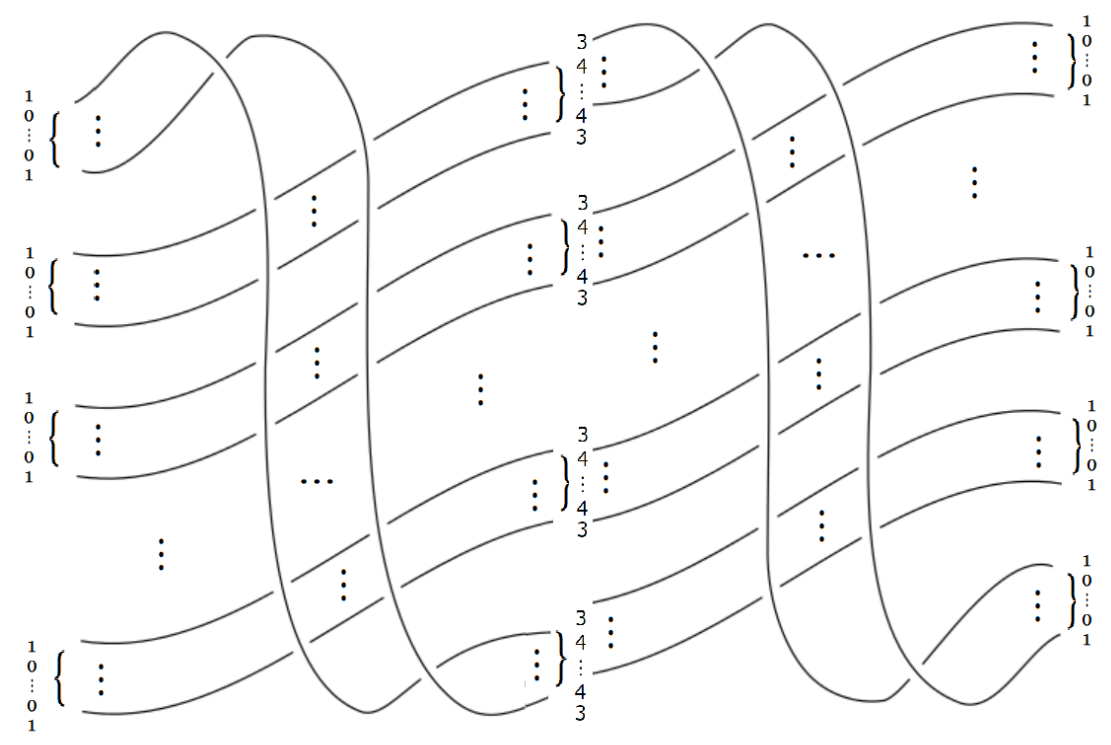

FiguRE 7.

That is, since $p$ is assumed to be even, $D$ admits a $\mathbb{Z}$-coloring which is composed by connecting $p / 2$ local diagrams illustrated by Figure 7 . It concludes that the colors of this $\mathbb{Z}$-coloring are $\{0,1,2,3,4\}$, that is, the $\mathbb{Z}$-coloring is represented by five colors.

Next, suppose that $r$ is odd and $q$ is even. In this case, we start with setting

$$
\left(\gamma\left(x_{i, 1}\right), \gamma\left(x_{i, 2}\right), \ldots, \gamma\left(x_{i, r}\right)\right)= \begin{cases}(2,1, \ldots, 1,0) & \text { if } i \text { is odd } \\ (0,1, \ldots, 1,2) & \text { if } i \text { is even }\end{cases}
$$

See Figure 8 (left). 

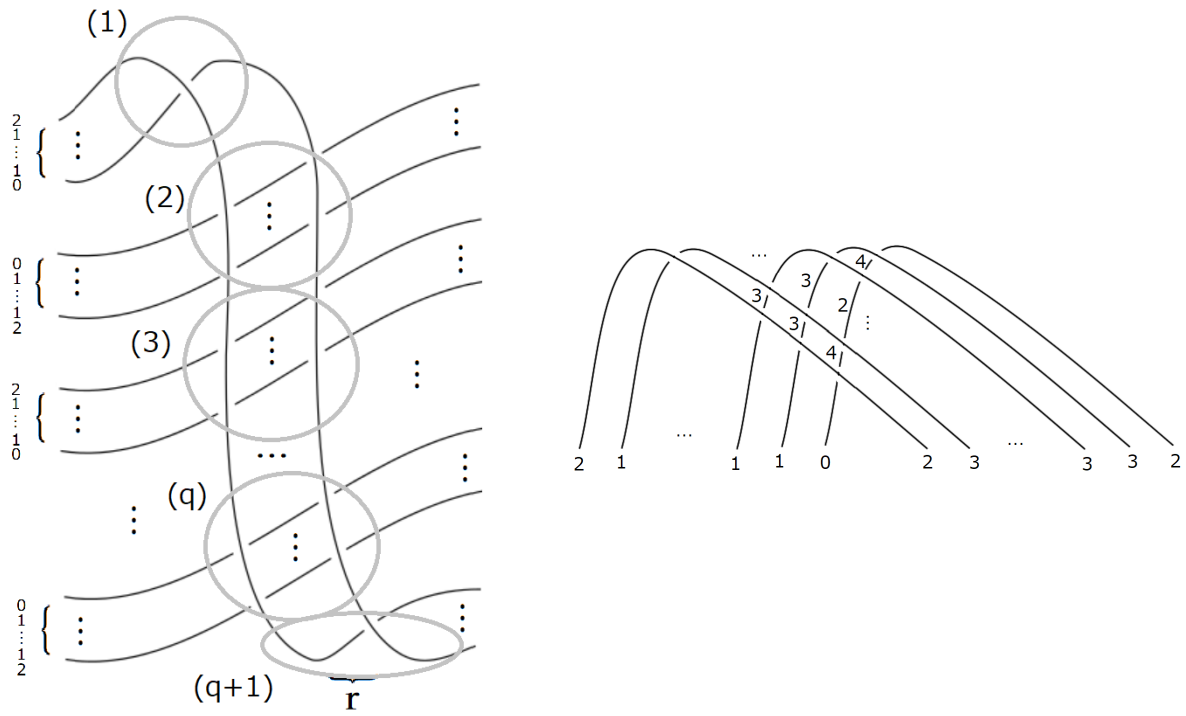

FIGURE 8.

As illustrated in Figure 8 (right), we can extend $\gamma$ on the arcs in the regions (1) and $(q+1)$ in Figure 2 (right). And, as illustrated in Figure 9, we can extend $\gamma$ on the arcs in the regions $(2),(3), \ldots,(q)$ in Figure 2
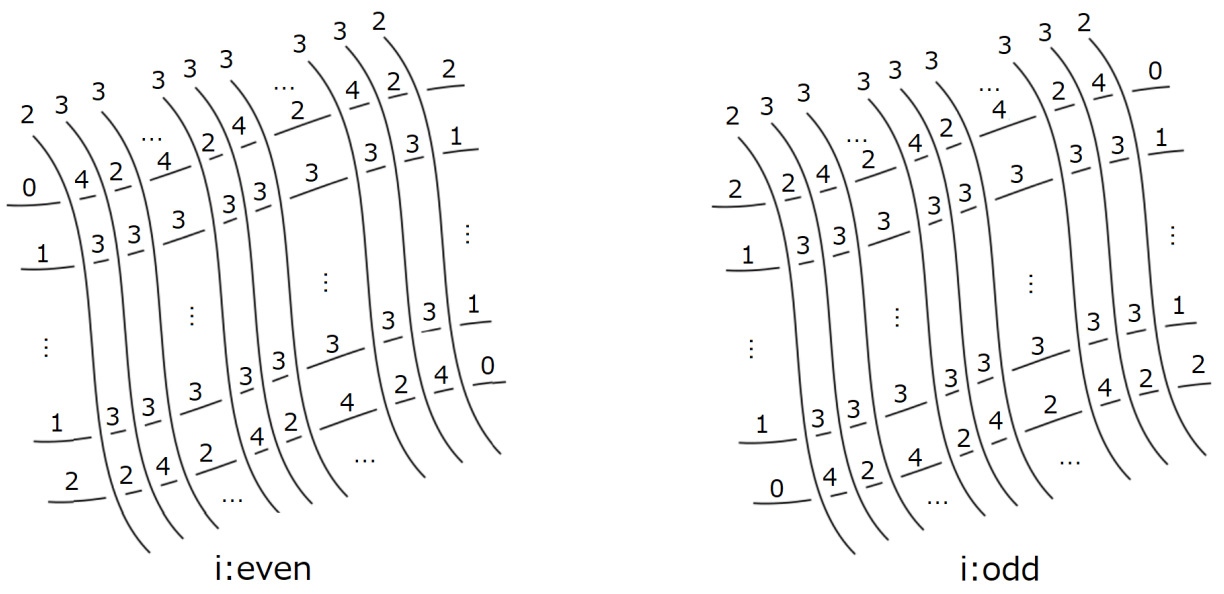

FiguRE 9.

Now, $\gamma$ can be extended on all the arcs in Figure 10. 


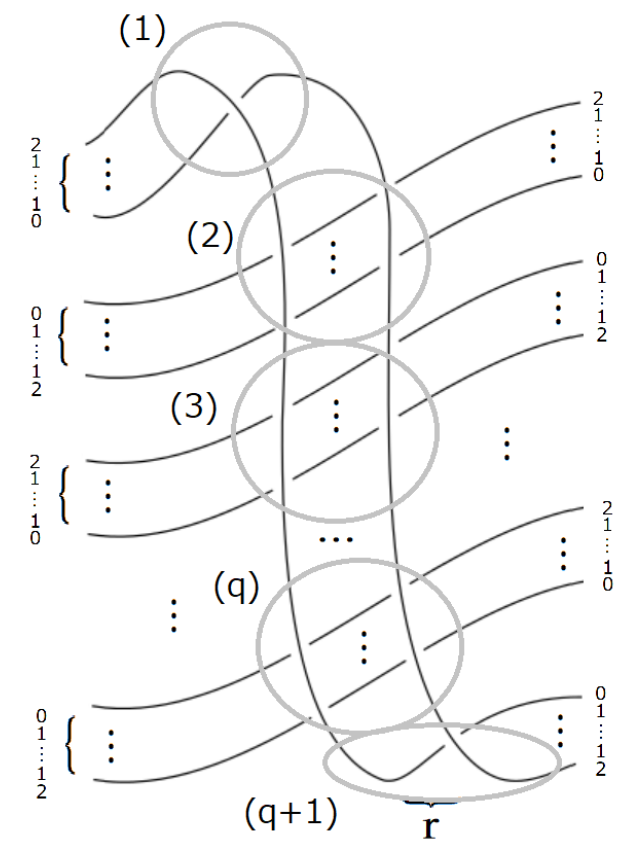

FigURE 10.

Since $D$ is composed of $p$ copies of the local diagram in Figure 2 it concludes that $D$ admits a $\mathbb{Z}$-coloring with only five colors $0,1,2,3$, and 4 .

Consequently, we obtain that $\operatorname{mincol}_{\mathbb{Z}}(D) \leq 5$.

We next show that the minimal coloring number $\operatorname{mincol}_{\mathbb{Z}}(D)$ of $D$ is at least five, i.e., $\operatorname{mincol}_{\mathbb{Z}}(D) \geq 5$.

Suppose for a contradiction that the diagram $D$ as shown in Figure 1 admits a non-trivial $\mathbb{Z}$-coloring $\gamma$ with only four colors. By [5, Theorem 3.2], we may assume that the image of $\gamma$ is $\{0,1,2,3\}$. By Lemma 3.2 we see that the over arcs are colored by 1 or 2 , otherwise $\gamma$ have to be trivial. Thus there exist only three ways to color a crossing:

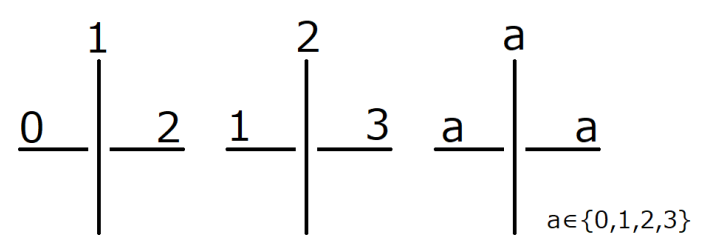

Figure 11. Colors at a crossing

Here we see that, in a component including an arc colored by 1 or 3 (resp. 0 or 2), the arcs are always colored by odd (resp. even) numbers, by the condition of the $\mathbb{Z}$-coloring. 
Then, since the number of the component is the odd number $r$, either the number of the components colored by odd numbers or that by even numbers is odd. Since the linear function $X \mapsto-X+3$ on $\mathbb{Z}$ switches these two cases, we only have to consider the former case; then the number of the over arcs colored by 1 is odd in the $r$ parallel over arcs as in Figure 12 .

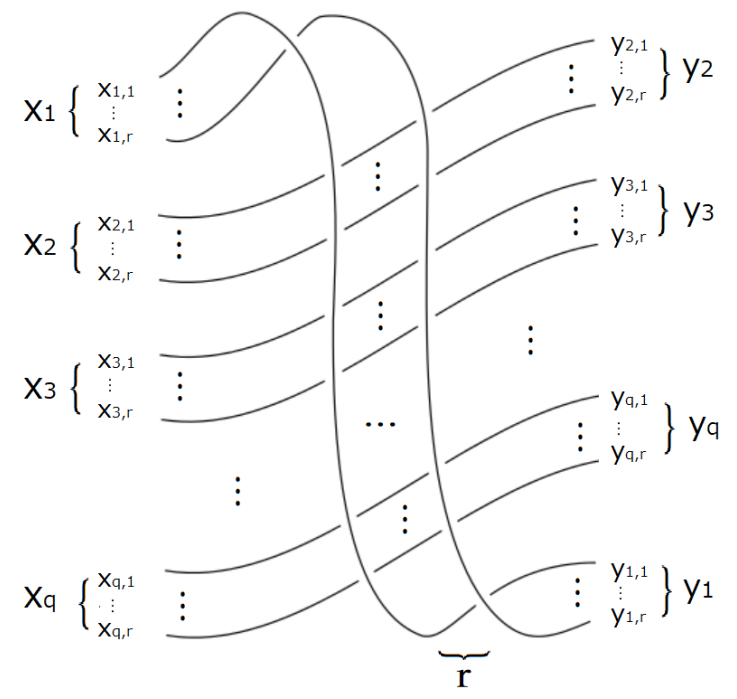

Figure 12.

In the case $q=1$, we consider the $r$ parallel arcs $\mathbf{x}=\left\{x_{1}, \ldots, x_{r}\right\}$ as shown in Figure 13

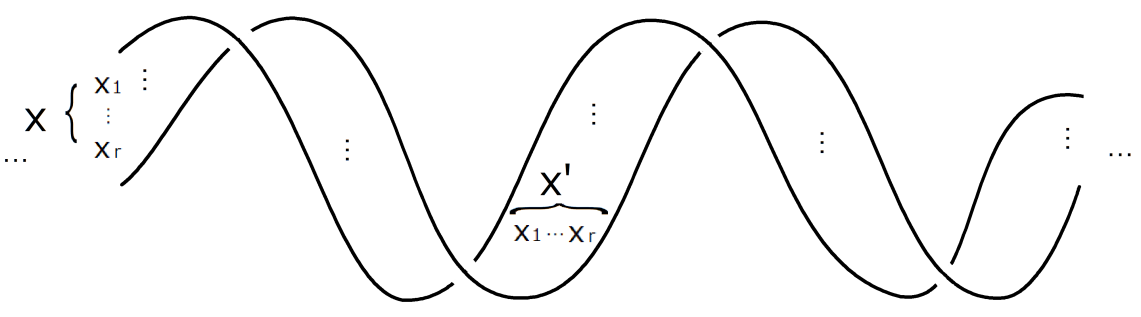

FigURE 13.

The colors on $\mathbf{x}$ is represented by $\gamma(\mathbf{x})=\left(\gamma\left(x_{1}\right), \ldots, \gamma\left(x_{r}\right)\right)$.

By the assumption that $\gamma$ is a non-trivial $\mathbb{Z}$-coloring, the diagram has an over arc colored by 2 . Hence we can label $\mathbf{x}$ to have $x_{r}$ be colored by 2 . That is, we consider the case the colors of $\mathbf{x}$ is $\gamma(\mathbf{x})=\left(a_{1}, a_{2}, \ldots, a_{r-1}, 2\right)$,

The arc $x_{r}$ turns into $x_{r}^{\prime}$ by passing through the odd arcs colored by 1 and even arcs colored by 2 . Here, as the diagram has exactly four colors $0,1,2,3$, the $\operatorname{arc} x_{r}^{\prime}$ is colored by 0 . That is, there exists an over arc colored by 0 as shown in Figure 14 


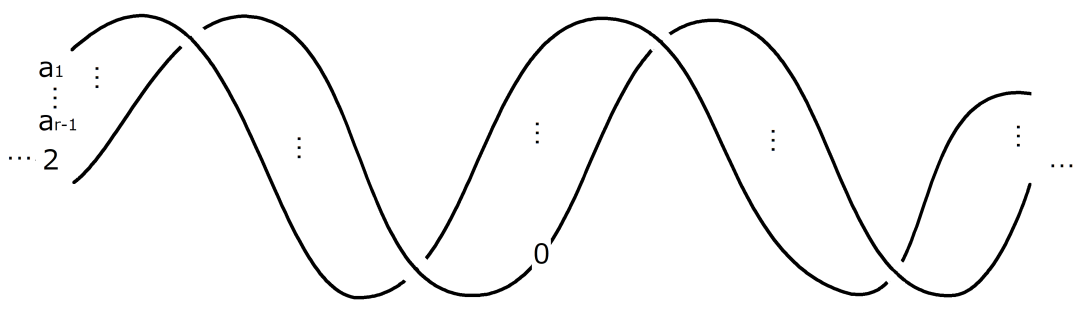

Figure 14.

This is a contradiction; the colors on over arcs are 1 or 2 .

In the case $q \geq 2$, We divide $q r$ parallel arcs into $q$ subfamilies $\mathbf{x}_{1}, \ldots, \mathbf{x}_{q}$ and $\mathbf{y}_{1}, \ldots, \mathbf{y}_{q}$ as depicted in Figure 12 . The colors on $\mathbf{x}_{i}=\left(x_{i, 1}, \ldots, x_{i, r}\right)$ are represented by $\gamma\left(\mathbf{x}_{i}\right)=\left(\gamma\left(x_{i, 1}\right), \ldots, \gamma\left(x_{i, r}\right)\right)$.

By the condition of a $\mathbb{Z}$-coloring, the colors on $\mathbf{y}_{i}$ are expressed by using a linear function $f$ as $\gamma\left(\mathbf{y}_{i}\right)=\left\{f\left(\gamma\left(x_{i, 1}\right)\right), \ldots, f\left(\gamma\left(x_{i, r}\right)\right)\right\}$ with $i=2,3, \ldots, q$. Then we see $f(0)=2$ and $f(2)=0$, as the number of the over arcs colored by 1 is odd in the $r$ over arcs in Figure 12 .

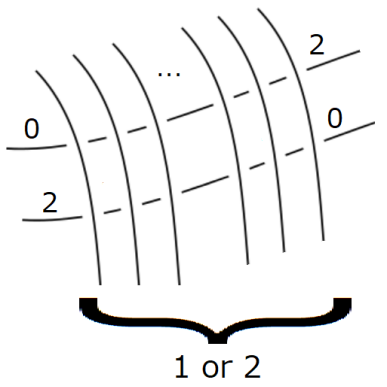

FIGURE 15.

Here we obtain $f(X)=-X+2$ and see $f(3)=-1$. That is, if there exists an arc colored by 3 in any parallel $\operatorname{arcs} \mathbf{x}_{i}(i=2, \ldots, q)$, there exists an arc colored by -1 in the parallel arcs $\mathbf{y}_{i}$. It is a contradiction since the image of $\gamma$ is $\{0,1,2,3\}$.

By the assumption, the diagram has an arc colored by 3 . If there exist no arcs colored by 3 in parallel $\operatorname{arcs} \mathbf{x}_{i}$, we relabel the arcs to have the arc colored by 3 be one of parallel arcs. Furthermore, if there exist an arc colored by 3 in $\mathbf{x}_{1}$, we see the outside of the local diagram as shown in Figure 12 and we relabel to have $\mathbf{x}_{1}$ as $\mathbf{y}_{1}$. Since $f$ is an involution, this completes the theorem.

\section{4. $\mathbb{Z}$-COLORINGS OF TORUS LINK DIAGRAMS}

In this section, we give complete classifications of all $\mathbb{Z}$-colorings of the standard diagram of $T(a, b)$ (Proposition 4.3) and of all $\mathbb{Z}$-colorings by only four colors of $T(a, b)$ (Proposition 4.4). 
To achieve these, we prepare a theorem on $\mathbb{Z}$-colorings of $n$-parallels of knots. For a knot diagram $D$, we obtain another diagram $D^{(n)}$ by replacing the string with $n$ parallel copies of it; we call $D^{(n)}$ the $n$-parallel of $D$. Remark that $D^{(n)}$ represents the $(n w, n)$-cable link of the knot represented by $D$, where $w$ is the writhe of $D$. In the following, we denote the set of $\mathbb{Z}$-colorings of a link diagram $D$ by $\mathrm{Col}_{\mathbb{Z}}(D)$.

Theorem 4.1. Let $D$ be an oriented knot diagram and $D^{(n)}$ the $n$-parallel of $D$. We fix any arc of $D$ and let $\gamma_{1}, \ldots, \gamma_{n}$ be the corresponding $n$ arcs of $D^{(n)}$. We define a homomorphism $r: \operatorname{Col}_{\mathbb{Z}}\left(D^{(n)}\right) \rightarrow \mathbb{Z}^{n}$ as

$$
r(\mathcal{C})=\left(\mathcal{C}\left(\gamma_{1}\right), \ldots, \mathcal{C}\left(\gamma_{n}\right)\right) \text {. }
$$

Then, $r$ is injective and the image $\operatorname{Im} r$ of $r$ is equal to

$$
\begin{cases}\left\{\left(a_{1}, \ldots, a_{n}\right) \mid w\left(a_{1}-a_{2}+a_{3}-\cdots-a_{n}\right)=0\right\} & \text { if } n \text { is even, } \\ \{(a, \ldots, a) \mid a \in \mathbb{Z}\} & \text { if } n \text { is odd and } w \text { is odd, } \\ \mathbb{Z}^{n} & \text { if } n \text { is odd and } w \text { is even }\end{cases}
$$

where $w$ is the writhe of $D$.

Remark 4.2. Theorem 4.1 states that the colors $a_{1}, \ldots, a_{n}$ of the $n$-paralleled arcs of an arc $\gamma$ determine the whole coloring, especially the colors $a_{1}^{\prime}, \ldots, a_{n}^{\prime}$ of the $n$-parallel of another arc $\gamma^{\prime}$. As we see in the proof, we can calculate $a_{1}^{\prime}, \ldots, a_{n}^{\prime}$ from a cyclic-rack coloring of $D$. For example, if $n$ is even and $w \neq 0$, we always have $a_{i}^{\prime}=a_{i}$ for any $i$. If $n$ is odd and $w$ is even, we track the string of $D$ from $\gamma$ to $\gamma^{\prime}$ and count the number of times of passing under arcs; if it is even, then $a_{i}^{\prime}=a_{i}$, and otherwise, $a_{i}^{\prime}=-a_{i}+2\left(a_{1}-a_{2}+\cdots+a_{n}\right)$.

We include a proof of Theorem 4.1 in Appendix, for it is rather independent from the other contents of the paper.

4.1. Determining $\mathbb{Z}$-colorings. Let $B(a, b)$ denote the braid (diagram) illustrated in Figure 16. We can regard the torus link $T(a, b)$ as the closure of $B(a, b)$. Then, an assignment of colors to the $b$ left ends of $B(a, b)$ determines a $\mathbb{Z}$-coloring of $B(a, b)$, and if the resulting colors of the right ends coincide with the left colors, it gives a $\mathbb{Z}$-coloring of the standard diagram of $T(a, b)$.

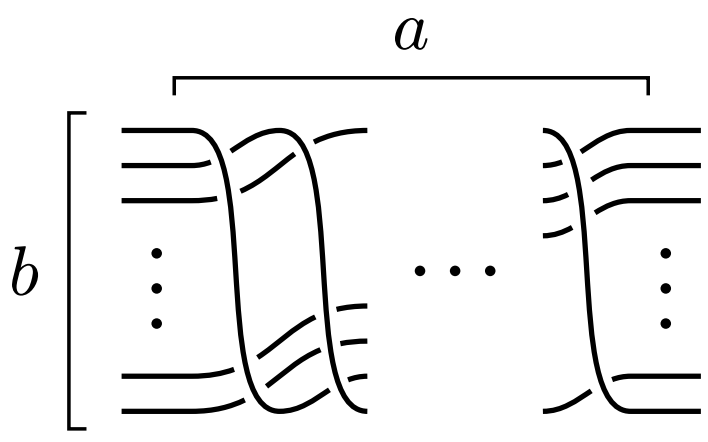

Figure 16. $B(a, b)$

Let $p, q$, and $r$ be nonzero integers such that $|p| \geq q \geq 1$ and $r \geq 2$, and assume that $p$ and $q$ are mutually prime. We divide the $q r$ arcs of the left end of $B(p r, q r)$ 
into $q$ subfamilies $\boldsymbol{x}_{1}, \ldots, \boldsymbol{x}_{q}$ as depicted in Figure 2 (left). Let $A$ be the set of the $q r$-tuples of integers which give a $\mathbb{Z}$-coloring of the standard diagram $D$ of $T(p r, q r)$, i.e.,

$$
A=\left\{\left(\boldsymbol{a}_{1}, \ldots, \boldsymbol{a}_{q}\right) \in\left(\mathbb{Z}^{r}\right)^{q} \mid \begin{array}{l}
\text { the assignment of } \boldsymbol{a}_{1}, \ldots, \boldsymbol{a}_{q} \in \mathbb{Z}^{r} \text { to } \boldsymbol{x}_{1}, \ldots, \boldsymbol{x}_{q} \\
\text { defines a } \mathbb{Z} \text {-coloring of } D
\end{array}\right\} .
$$

The following proposition describes the coloring of torus links:

Proposition 4.3. We have

$$
A= \begin{cases}\left\{(\boldsymbol{a}, \ldots, \boldsymbol{a}) \mid \boldsymbol{a} \in \mathbb{Z}^{r}, \Delta(\boldsymbol{a})=0\right\} & \text { if } r \text { is even, } \\ \left\{(\boldsymbol{a}, \ldots, \boldsymbol{a}) \mid \boldsymbol{a} \in \mathbb{Z}^{r}\right\} & \text { if } r \text { is odd and } p \text { is even, } \\ \left\{(\boldsymbol{a}, \tau(\boldsymbol{a}), \boldsymbol{a}, \ldots, \tau(\boldsymbol{a})) \mid \boldsymbol{a} \in \mathbb{Z}^{r}\right\} & \text { if } r \text { is odd and } q \text { is even, }\end{cases}
$$

where $\Delta(\boldsymbol{a})=a_{1}-a_{2}+\cdots+(-1)^{r} a_{r} \in \mathbb{Z}$ and $\tau(\boldsymbol{a})=\left(-a_{i}+2 \Delta(\boldsymbol{a})\right)_{i} \in \mathbb{Z}^{r}$ for $\boldsymbol{a}=\left(a_{1}, \ldots, a_{r}\right) \in \mathbb{Z}^{r}$.

Proof. Let $B_{0}$ be the tangle diagram depicted in Figure 17 and $B_{0}^{(r)}$ the $r$-parallel of $B_{0}$. We denote the closures of $B_{0}$ and $B_{0}^{(r)}$ by $D_{0}$ and $D_{0}^{(r)}$, respectively. Since $D_{0}$ represents the knot $T(p, q)$, we can apply Theorem 4.1 to $D_{0}$ and its $r$-parallel $D_{0}^{(r)}$ to determine the colorings of $D_{0}^{(r)}$. Furthermore, we should remark that $B_{0}^{(r)}$ and $B(p r, q r)$ are isotopic; we find the colorings of $D$ from those of $D_{0}^{(r)}$, using an isotopy which takes $D_{0}^{(r)}$ to $D$ and fixes the $\operatorname{arcs} x_{i, j}(1 \leq i \leq q, 1 \leq j \leq r)$ and their colors.

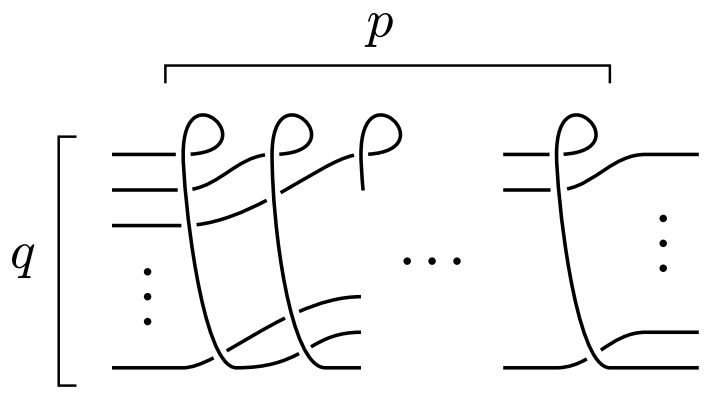

Figure 17. $B_{0}$

By Theorem 4.1 (and Remark 4.2 , a coloring of $D_{0}^{(r)}$ is determined by the color $\boldsymbol{a} \in \mathbb{Z}^{r}$ of $r \operatorname{arcs} \boldsymbol{x}_{1}$, and the whole coloring is found from a cyclic-rack coloring of $D_{0}$. If $r$ is even, the color $\boldsymbol{a}$ has to satisfy the condition $\Delta(\boldsymbol{a})=0$ and then the color of $\boldsymbol{x}_{i}$ is equal to $\boldsymbol{a}$ for any $i$. In the other cases, we can choose any $\boldsymbol{a} \in \mathbb{Z}^{r}$, and we consider the cyclic rack $C_{2}$ of order 2 to examine the whole coloring. A brief calculation finds a $C_{2}$-coloring of $D_{0}$, which colors the left $q$ arcs

$$
\begin{cases}0,0,0,0, \ldots, 0 & \text { if } p \text { is even and } q \text { is odd, } \\ 0,1,0,1, \ldots, 0 & \text { if } p \text { is odd and } q \text { is even. }\end{cases}
$$

In terms of Remark 4.2 , a color $0 \in C_{2}$ corresponds to "even" and $1 \in C_{1}$ to "odd"; as in the remark, we associate $\boldsymbol{a}$ to the $r$-paralleled arcs of an arc if it has a color 0 , and associate $\tau(\boldsymbol{a})$ otherwise. This completes the proposition. 
4.2. Coloring with four colors. Let $p, q$, and $r$ be non-zero integers such that $p$ and $q$ are relatively prime, $|p| \geq q \geq 1$, and $r \geq 2$. By Theorem 1.1, the standard diagram $D$ of $T(p r, q r)$ admits a $\mathbb{Z}$-coloring $\gamma$ with four colors if and only if $r$ is even. In this case, it is sufficient to consider the case where $\operatorname{Im} \gamma=\{0,1,2,3\}$ by [5. Theorem 3.2]. Let $A^{(4)}$ be the set of the $q r$-tuples which give such $\mathbb{Z}$-colorings:

$A^{(4)}=\left\{\left(\boldsymbol{a}_{1}, \ldots, \boldsymbol{a}_{q}\right) \in\left(\mathbb{Z}^{r}\right)^{q} \mid \begin{array}{l}\text { the assignment of } \boldsymbol{a}_{1}, \ldots, \boldsymbol{a}_{q} \in \mathbb{Z}^{r} \text { to } \boldsymbol{x}_{1}, \ldots, \boldsymbol{x}_{q} \\ \text { defines a } \mathbb{Z} \text {-coloring of } D \text { with the four colors }\{0,1,2,3\}\end{array}\right\}$.

Here, we regard $D$ as the closure of $B(p r, q r)$ and denote the subfamilies of the left $q r$ arcs by $\boldsymbol{x}_{1}, \ldots, \boldsymbol{x}_{q}$ as shown in Figure 2 (left).

Proposition 4.4. We have

$$
A^{(4)}=\left\{(\boldsymbol{a}, \ldots, \boldsymbol{a}) \mid \boldsymbol{a} \in A_{01}^{(4)} \cup A_{12}^{(4)} \cup A_{23}^{(4)}\right\} \backslash\{(1, \ldots, 1),(2, \ldots, 2)\},
$$

where

$$
\begin{aligned}
& A_{01}^{(4)}=\left\{\left(a_{1}, \ldots, a_{r}\right) \in\{0,1\}^{r} \mid a_{1}=a_{r}=1, a_{2 i}=a_{2 i+1}(i=1, \ldots, r / 2-1)\right\}, \\
& A_{12}^{(4)}=\left\{\left(a_{1}, \ldots, a_{r}\right) \in\{1,2\}^{r} \mid a_{2 i-1}=a_{2 i}(i=1, \ldots, r / 2)\right\}, \\
& A_{23}^{(4)}=\left\{\left(a_{1}, \ldots, a_{r}\right) \in\{2,3\}^{r} \mid a_{1}=a_{r}=2, a_{2 i}=a_{2 i+1}(i=1, \ldots, r / 2-1)\right\} .
\end{aligned}
$$

Proof. By Proposition 4.3 a member of $A^{(4)}$ is expressed as $(\boldsymbol{a}, \ldots, \boldsymbol{a})$, where $\boldsymbol{a}=\left(a_{1}, \ldots, a_{r}\right)$ is an $r$-tuple of integers with $\Delta(\boldsymbol{a})=a_{1}-a_{2}+\cdots-a_{r}$ equal to 0. We have to show that $\boldsymbol{a} \in A_{01}^{(4)} \cup A_{12}^{(4)} \cup A_{23}^{(4)}$.

Let $S$ be the set $\left\{a_{1}, \ldots, a_{r}\right\}$. We first assert that $S=\{0,1\},\{1,2\}$, or $\{2,3\}$. To show $S \not \supset\{0,2\}$, we suppose that $\left\{a_{i}, a_{j}\right\}=\{0,2\}(i<j)$. The $\operatorname{arc} x_{1, i}$ goes under the $i-1$ arcs to become an over arc $x_{i}^{\prime}$ with color $a_{i}^{\prime}$, whereas $x_{1, j}$ goes under the same arcs to become $x_{j}^{\prime}$ with color $a_{j}^{\prime}$ and then passes under $x_{i}^{\prime}$. Here, we should remark that $\left|a_{i}^{\prime}-a_{j}^{\prime}\right|=2$ since $x_{1, i}$ and $x_{1, j}$ goes under the same arcs, but such a crossing is not allowed, as seen in the proof of Theorem 3.1. Thus we have $S \not \supset\{0,2\}$, and in the same way, we can check that $S \not \supset\{1,3\},\{0,3\}$. Therefore $S$ is a set of two consecutive integers, as asserted.

In the case where $S=\{1,2\}$, we see that $a_{1}=a_{2}$; otherwise, the color $-a_{2}+2 a_{1}$ of the over arc next to $x_{1,1}$ would not be 1 or 2 . After two twists, the colors of the arcs shift cyclically to be $\left(a_{3}, a_{4}, \ldots, a_{2}\right)$ since passing under two strings with a same color does not change the color. Then, the argument above shows that $a_{3}=a_{4}$. Repeating this, we find $a_{2 i-1}=a_{2 i}$ for $i=1, \ldots, r / 2$, i.e., $\boldsymbol{a} \in A_{12}^{(4)}$.

In the case where $S=\{0,1\}$ (resp. $\{2,3\}$ ), the colors $a_{1}$ and $a_{r}$ of over $\operatorname{arcs}$ have to be 1 (resp. 2). After $x_{1,1}$ comes to the bottom, the colors of the arcs are $\left(-a_{2}+2 a_{1},-a_{3}+2 a_{1}, \ldots,-a_{r}+2 a_{1}, a_{1}\right)$, and this is also a member of $A^{(4)}$. Since the set $\left\{a_{1},-a_{2}+2 a_{1}, \ldots,-a_{r}+2 a_{1}\right\}$ is equal to $\{1,2\}$, we have $a_{2 i}=a_{2 i+1}(i=$ $1, \ldots, r / 2-1)$ as shown above. This means that $\boldsymbol{a} \in A_{01}^{(4)}\left(\right.$ resp. $\left.A_{23}^{(4)}\right)$.

Let $A^{\prime}$ be the set $\left\{(\boldsymbol{a}, \ldots, \boldsymbol{a}) \mid \boldsymbol{a} \in A_{01}^{(4)} \cup A_{12}^{(4)} \cup A_{23}^{(4)}\right\} \backslash\{(1, \ldots, 1),(2, \ldots, 2)\}$. We have proven that $A^{(4)} \subset A^{\prime}$ as above. A brief calculation shows that $\Delta(\boldsymbol{a})=0$ for $\boldsymbol{a} \in A_{01}^{(4)} \cup A_{12}^{(4)} \cup A_{23}^{(4)}$ and hence $(\boldsymbol{a}, \ldots, \boldsymbol{a}) \in A^{\prime}$ gives a $\mathbb{Z}$-coloring of $D$. To see that every element of $A^{\prime}$ actually defines a four-color coloring, it is sufficient to show that for any $\left(a_{1}, \ldots, a_{q r}\right) \in A^{\prime},\left(-a_{2}+2 a_{1}, \ldots,-a_{q r}+2 a_{1}, a_{1}\right)$ is also a member of $A^{\prime}$; this means that "one twist preserves $A^{\prime \prime}$ ", and then implies that there 
are only four colors in the resultant $\mathbb{Z}$-coloring. This is verified by the definitions of $A_{01}^{(4)}, A_{12}^{(4)}$, and $A_{23}^{(4)}$. Thus we have $A^{\prime} \subset A^{(4)}$ as required.

\section{REFERENCES}

[1] N. Andruskiewitsch and M. Graña, From racks to pointed Hopf algebras, Adv. Math. 178 (2003), no. 2, 177-243.

[2] M. Elhamdadi and S. Nelson, Quandles - an introduction to the algebra of knots, Student Mathematical Library, 74, American Mathematical Society, Providence, RI, 2015.

[3] R. H. Fox, A quick trip through knot theory, in Topology of 3-manifolds and related topics (Proc. The Univ. of Georgia Institute, 1961), 120-167, Prentice-Hall, Englewood Cliffs, NJ.

[4] A. Kawauchi, Lectures on knot theory, Monograph in Japanese, 2007, Kyoritsu Shuppan Co. Ltd.

[5] K. Ichihara and E. Matsudo, Minimal coloring number for $\mathbb{Z}$-colorable links, J. Knot Theory Ramifications 26 (2017), no. 4, 1750018, 23 pp.

[6] K. Ichihara and E. Matsudo, Minimal coloring number on minimal diagrams for $\mathbb{Z}$-colorable links, Proc. Inst. Nat. Sci., Nihon Univ. 53 (2018), 231-237. https://www.chs.nihonu.ac.jp/institute/nature/kiyou/2018/53/16.pdf

[7] K. Ishikawa, Cabling formulae of quandle cocycle invariants for surface knots, Master thesis, Kyoto University, January, 2016.

[8] E. Matsudo, Minimal coloring number for $\mathbb{Z}$-colorable links II, J. Knot Theory Ramifications 28 (2019), no. 7, 1950047.

[9] T. Naruse, Kyokumen-musubime no quandle cocycle fuhenryo no tajuka-koshiki (Paralleledversions of quandle cocycle invariants for surface knots (in Japanese)), Master thesis, Kyoto University, January, 2015. http://hdl.handle.net/2433/194277

[10] M. Zhang, X. Jin and Q. Deng, The minimal coloring number of any non-splittable $\mathbb{Z}$-colorable link is four, J. Knot Theory Ramifications 26 (2017), no. 13, 1750084, 18 pp.

\section{APPENDIX A. $\mathbb{Z}$-COLORING OF $n$-PARALLELS OF KNOTS}

In this appendix, we give a proof of Theorem 4.1. In this proof, we use ideas of racks and quandles. For definitions of racks, quandles, and rack (quandle) colorings, see 2 for example. For a rack $R$, we denote the set of $R$-colorings of an oriented knot diagram $D$ by $\operatorname{Col}_{R}(D)$. For example, the set $\mathbb{Z}$ equipped with the binary operation $*$ defined by $a * b=2 b-a$ is a rack (it is in fact a quandle), and then $\mathrm{Col}_{\mathbb{Z}}(D)$ is the set of $\mathbb{Z}$-colorings as in Section 4

We denote the automorphism group of a rack $R=(R, *)$ by $\operatorname{Aut}(R, *)$. For each $a \in R$, the map $\bullet * a: R \ni x \mapsto x * a \in R$ is by definition is an automorphism of $R$, and then the subgroup of $\operatorname{Aut}(R, *)$ generated by $\bullet * a(a \in R)$ is called the inner automorphism group and denoted by $\operatorname{Inn}(R, *)$. We say that $R$ is (algebraically) connected if the action of $\operatorname{Inn}(R, *)$ on $R$ is transitive.

Furthermore, we should recall the quandles and kink maps associated to racks. The associated quandle $R_{Q}$ of a rack $R=(R, *)$ is the pair $\left(R, *_{Q}\right)$, where the binary operation $*_{Q}$ is defined by $x *_{Q} y=(x \bar{*} x) * y(x, y \in R)$, and the associated kink map $\tau$ is defined by $\tau(x)=x * x(x \in R)$. A brief calculation shows that $R_{Q}$ is a quandle and $\tau$ is a kink map of $R$ (see, e.g., [1]), i.e., $\tau$ is an automorphism of $R$ and for any $x, y \in R$ we have $x * \tau(y)=x * y$; this implies that $\tau$ is also a kink map of $R_{Q}$. We remark that the quandle $R_{Q}$ is equal to $R$ as a set, and an automorphism of $R$ is also an automorphism of $R_{Q}$; we may regard $\operatorname{Aut}(R, *)$ and $\operatorname{Inn}(R, *)$ as subgroups of $\operatorname{Aut}\left(R_{Q}\right)$.

To show the theorem, we introduce a rack $\mathbb{Z}_{R}^{n}$ as follows (this is due to [9]): for $\boldsymbol{x}=\left(x_{1}, \ldots, x_{n}\right), \boldsymbol{y}=\left(y_{1}, \ldots, y_{n}\right) \in \mathbb{Z}^{n}$, we put $\boldsymbol{x} *_{R} \boldsymbol{y}=\boldsymbol{z}=\left(z_{1}, \ldots, z_{n}\right)$, where

$$
z_{i}=\left(\left(\left(x_{i} * y_{1}\right) * y_{2}\right) \cdots\right) * y_{n} .
$$


By a brief calculation, we can check that $\mathbb{Z}_{R}^{n}=\left(\mathbb{Z}^{n}, *_{R}\right)$ is a rack.

In the proof of Theorem 4.1 below, we examine the $\mathbb{Z}_{R}^{n}$-colorings of the knot diagram $D$. Since the fundamental rack of a framed knot is connected, the image of a coloring of $D$ by a rack $R$ is contained in a connected subrack of $R$; if $R$ is decomposed into the maximal connected subracks $R_{\lambda}(\lambda \in \Lambda)$ (for the existence and uniqueness of the decomposition, see, e.g., [1]), we have $\operatorname{Col}_{R}(D)=\bigsqcup_{\lambda \in \Lambda} \operatorname{Col}_{R_{\lambda}}(D)$. The following lemma describes the subracks $R_{\lambda}$ when $R=\mathbb{Z}_{R}^{n}$.

Lemma A.1. Each maximal connected subrack of $\mathbb{Z}_{R}^{n}$ is a cyclic rack.

Recall that a cyclic rack $C_{k}\left(k \in \mathbb{Z}_{\geq 0}\right)$ is a cyclic group $\mathbb{Z} / k \mathbb{Z}$ with the binary operation $*$ defined by $a * b=a+1(a, b \in \mathbb{Z} / k \mathbb{Z})$. A rack isomorphic to a cyclic rack is also called a cyclic rack.

Proof of Lemma A.1. Let $\mathbb{Z}_{Q}^{n}=\left(\mathbb{Z}^{n}, *_{Q}\right)$ be the associated quandle of $\mathbb{Z}_{R}^{n}$ and $\tau$ the associated kink map. We first claim that each maximal connected subquandle of $\mathbb{Z}_{Q}^{n}$ is the trivial quandle of order 1 . In fact, a brief calculation shows that

$$
\left(x_{i}\right)_{i} *_{Q}\left(y_{i}\right)_{i}=\left(x_{i}+2\left(-x_{n}+x_{n-1}-\cdots+(-1)^{n} x_{1}+(-1)^{n+1} y_{1}+\cdots+y_{n}\right)\right)_{i},
$$

for $\left(x_{i}\right)_{i},\left(y_{i}\right)_{i} \in \mathbb{Z}_{Q}^{n}$. This shows that the orbit of $\left(x_{i}\right)_{i} \in \mathbb{Z}_{Q}^{n}$ under the action of $\operatorname{Inn}\left(\mathbb{Z}_{Q}^{n}\right)$ is included in $\left\{\left(x_{i}+a\right)_{i} \mid a \in \mathbb{Z}\right\}$. Furthermore, since

$\left(x_{i}+a\right)_{i} *_{Q}\left(x_{i}+b\right)_{i}=\left(x_{i}+a+\left(1-(-1)^{n}\right)(b-a)\right)_{i}=\left(x_{i}+(-1)^{n} a+\left(1-(-1)^{n}\right) b\right)_{i}$,

the quandle $\left\{\left(x_{i}+a\right)_{i} \mid a \in \mathbb{Z}\right\}$ is isomorphic to $\mathbb{Z}$ or a trivial quandle. In either case, each maximal connected subquandle is the trivial quandle of order 1 . The lemma follows from this claim and Lemma A.2 below.

Lemma A.2. Let $R=(R, *)$ be a rack, $R_{Q}=\left(R, *_{Q}\right)$ the associated quandle, and $\tau$ the associated kink map. We denote the maximal connected subrack of $R$ (resp. $\left.R_{Q}\right)$ containing $x \in R\left(\right.$ resp. $\left.R_{Q}\right)$ by $\mathcal{M}_{x}$ (resp. $\left.\mathcal{M}_{x}^{Q}\right)$. Then we have $\mathcal{M}_{x}=\bigcup_{m \in \mathbb{Z}} \tau^{m}\left(\mathcal{M}_{x}^{Q}\right)$.

Proof. We set $\mathcal{M}_{x}^{\prime}=\bigcup_{m \in \mathbb{Z}} \tau^{m}\left(\mathcal{M}_{x}^{Q}\right)$. We have

$$
\begin{aligned}
\tau^{m}(y) * \tau^{n}(z) & =\left(\left(\tau^{m}(y) * \tau^{m}(y)\right) \bar{*} \tau^{m}(y)\right) * z=\tau^{m+1}(y) *_{Q} z \\
& =\tau^{m+1}\left(y *_{Q} z\right)
\end{aligned}
$$

and similarly $\tau^{m}(y) * \tau^{n}(z)=\tau^{m-1}\left(y \overline{*_{Q}} z\right)$ for any $y, z \in R$ (especially for $y, z \in \mathcal{M}_{x}^{Q}$ ) and $m, n \in \mathbb{Z}, \mathcal{M}_{x}^{\prime}$ is a connected subrack of $R$; hence we have $\mathcal{M}_{x}^{\prime} \subset \mathcal{M}_{x}$. Furthermore, by the definitions of $*_{Q}$ and $\tau, \operatorname{Inn}\left(\mathcal{M}_{x}, *\right)$ is contained in the subgroup of $\operatorname{Aut}\left(\mathcal{M}_{x}, *_{Q}\right)$ generated by $\operatorname{Inn}\left(\mathcal{M}_{x}, *_{Q}\right)$ and $\tau$. Since $\tau$ is central in this subgroup (recall that $\tau$ is a kink map of $\left(\mathcal{M}_{x}, *_{Q}\right)$, we have $\mathcal{M}_{x}=\bigcup_{m} \tau^{m}\left(\mathcal{M}_{x}^{\prime Q}\right)$, where we set $\mathcal{M}_{x}^{\prime Q}=\operatorname{Inn}\left(\mathcal{M}_{x}, *_{Q}\right) \cdot x$, i.e., the orbit of $x$ under the action of $\operatorname{Inn}\left(\mathcal{M}_{x}, *_{Q}\right)$. Here, the inner automorphism group $\operatorname{Inn}\left(\mathcal{M}_{x}, *_{Q}\right)$ is by definition generated by $\bullet *_{Q} y\left(y \in \mathcal{M}_{x}\right)$, but for each $y \in \mathcal{M}_{x}=\bigcup_{m} \tau^{m}\left(\mathcal{M}_{x}^{\prime Q}\right)$ there exists $y^{\prime} \in \mathcal{M}_{x}^{\prime Q}$ such that $\bullet *_{Q} y=\bullet *_{Q} y^{\prime}$; in fact, if $y \in \tau^{m}\left(\mathcal{M}_{x}^{\prime Q}\right)$, we can take $\tau^{-m}(y)$ as $y^{\prime}$. Thus, $\mathcal{M}_{x}^{\prime Q}=\operatorname{Inn}\left(\mathcal{M}_{x}^{\prime Q}, *_{Q}\right) \cdot x$, which implies that $\mathcal{M}_{x}^{\prime Q}$ is a connected subquandle. Then we have $\mathcal{M}_{x}^{\prime Q} \subset \mathcal{M}_{x}^{Q}$ and hence $\mathcal{M}_{x}=\bigcup_{m} \tau^{m}\left(\mathcal{M}_{x}^{\prime Q}\right) \subset$ $\bigcup_{m} \tau^{m}\left(\mathcal{M}_{x}^{Q}\right)=\mathcal{M}_{x}^{\prime}$, as required. 

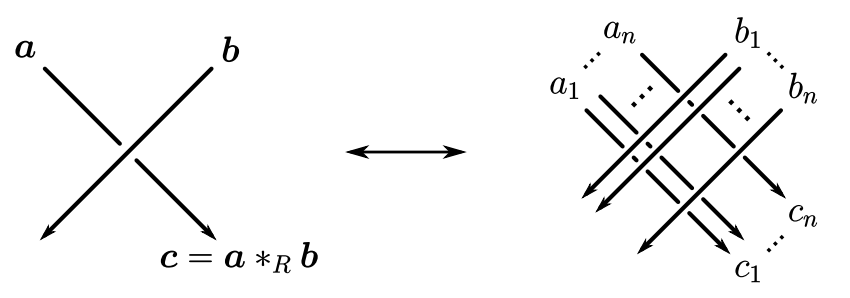

FiguRE 18. A bijection between $\operatorname{Col}_{\mathbb{Z}^{n}}(D)$ and $\operatorname{Col}_{\mathbb{Z}}\left(D^{(n)}\right)$

Proof of Theorem 4.1. Given a $\mathbb{Z}_{R}^{n}$-coloring $\mathcal{C}^{(n)}=\left(\mathcal{C}_{1}, \ldots, \mathcal{C}_{n}\right):\{\operatorname{arcs}\} \rightarrow \mathbb{Z}_{R}^{n}$ on $D$, we put colors $\mathcal{C}_{1}(\alpha), \ldots, \mathcal{C}_{n}(\alpha)$ to the $n$ arcs of $D^{(n)}$ corresponding to each $\operatorname{arc} \alpha$ of $D$. This is uniquely extended to be a whole coloring on $D^{(n)}$ (see Figure 18). Conversely, a $\mathbb{Z}$-coloring $\mathcal{C}$ of $D^{(n)}$ defines a $\mathbb{Z}_{R}^{n}$ coloring: we associate $\left(\mathcal{C}\left(\alpha_{1}\right), \ldots, \mathcal{C}\left(\alpha_{n}\right)\right)$ to an $\operatorname{arc} \alpha$ of $D$, where $\alpha_{1}, \ldots, \alpha_{n}$ are the $n$ arcs of $D^{(n)}$ corresponding to $\alpha$; by the definition of $*_{R}$, this defines a $\mathbb{Z}_{R}^{n}$-coloring. Thus, we have a bijection between $\mathrm{Col}_{\mathbb{Z}^{n}}(D)$ and $\mathrm{Col}_{\mathbb{Z}}\left(D^{(n)}\right)$. In the following, we identify these two sets by this bijection.

By Lemma A.1, any $\mathbb{Z}_{R}^{n}$-coloring on $D$ is a coloring by a subrack, which is a cyclic rack. Since we only have to consider cyclic-rack colorings, a given color $\boldsymbol{a} \in \mathbb{Z}_{R}^{n}$ on the fixed arc $\gamma$ uniquely determines the colors of the other arcs successively, and it defines a whole coloring if and only if they accords when we go back to $\gamma$, i.e., $\tau^{w}(\boldsymbol{a})=\boldsymbol{a}$, where $\tau$ is the associated kink map. Thus, $r$ is injective and $\operatorname{Im} r=\left\{\boldsymbol{a} \in \mathbb{Z}^{n} \mid \tau^{w}(\boldsymbol{a})=\boldsymbol{a}\right\}$.

By a concrete calculation we find that

$$
\tau(\boldsymbol{a})=\left((-1)^{n} a_{i}+2\left(a_{n}-a_{n-1}+\cdots+(-1)^{n-1} a_{1}\right)\right)_{i} .
$$

If $n$ is even, this shows that

$$
\tau^{w}(\boldsymbol{a})=\left(a_{i}+2 w\left(a_{n}-a_{n-1}+\cdots-a_{1}\right)\right)_{i} .
$$

Then $\tau^{w}(\boldsymbol{a})=\boldsymbol{a}$ if and only if $w\left(a_{1}-a_{2}+\cdots-a_{n}\right)=0$, as required. Next, suppose that $n$ is odd. In this case, a brief calculation shows that $\tau^{2}=\mathrm{id}_{\mathbb{Z}^{n}}$. Then, if $w$ is even, the condition $\tau^{w}(\boldsymbol{a})=\boldsymbol{a}$ is always satisfied. If $w$ is odd, $\tau^{w}(\boldsymbol{a})(=\tau(\boldsymbol{a}))$ equals $\boldsymbol{a}$ if and only if $a_{i}=-a_{i}+2 \Delta$ for $i=1, \ldots, n$, where $\Delta=a_{n}-a_{n-1}+\cdots+a_{1}$. This implies that $a_{i}=\Delta$ for any $i$ and hence $a_{1}=\cdots=a_{n}$; this concludes the theorem.

Department of Mathematics, College of Humanities and Sciences, Nihon University, 3-25-40 Sakurajosui, Setagaya-ku, Tokyo 156-8550, Japan

E-mail address: ichihara.kazuhiro@nihon-u.ac.jp

Research Institute for Mathematical Sciences, Kyoto University, Kyoto 606-8502, JAPAN

E-mail address: katsumi@kurims.kyoto-u.ac.jp

Institute of Natural Sciences, Nihon University, 3-25-40 Sakurajosui, Setagaya-ku, TOKYO 156-8550, JAPAN

E-mail address: cher16001@g.nihon-u.ac.jp 